\title{
Meroterpenoids from Ganoderma Species: A Review of Last Five Years
}

\author{
Xingrong Peng ${ }^{1} \cdot$ Minghua Qiu ${ }^{1,2}$
}

Received: 10 April 2018/Accepted: 23 April 2018/Published online: 2 May 2018

(C) The Author(s) 2018

\begin{abstract}
Meroterpenoids are hybrid natural products that partially originate from the terpenoid pathway. Ganoderma meroterpenoids (GMs) are a type of meroterpenoids containing a 1,2,4-trisubstituted phenyl and a polyunsaturated terpenoid part. Over last 5 years, great efforts have been made to conduct phytochemistry research on the genus Ganoderma, which have led to the isolation and identification of a number of GMs. These newly reported GMs showed diverse structures and a wide range of biological activities. This review gives an overview of new GMs from genus Ganoderma and their biological activities and biosynthetic pathway, focusing on the period from 2013 until 2018.
\end{abstract}

\section{Graphical Abstract}

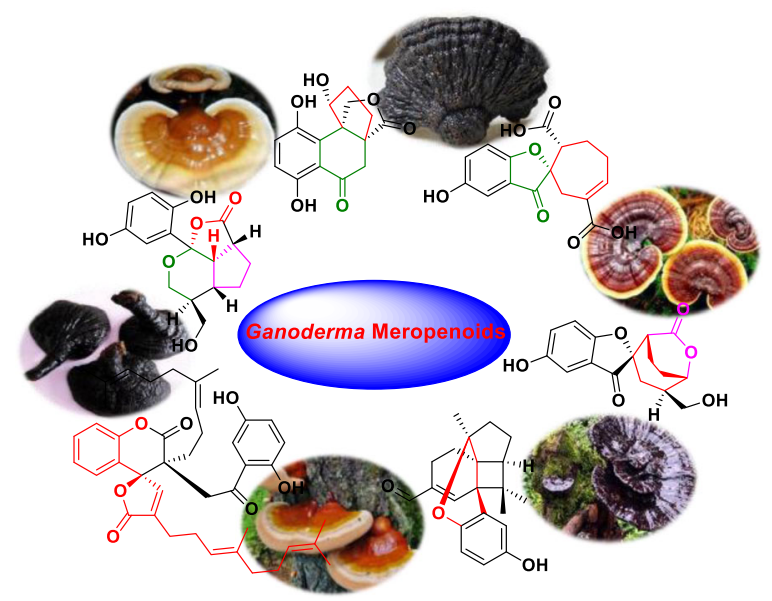

Keywords Ganoderma $\cdot$ Ganoderma meroterpenoids $\cdot$ New structures $\cdot$ Biological activities

\section{Introduction}

Ganoderma is a ganodermataceae (basidiomycete) white rot fungus, normally growing on woody plants and wood $\operatorname{logs}[1]$, and is used for medicinal purposes in China,

Minghua Qiu

mhchiu@mail.kib.ac.cn

1 State Key Laboratory of Phytochemistry and Plant Sources in West China, Kunming Institute of Botany, Chinese Academy of Sciences, Kunming 650201, People's Republic of China

2 University of the Chinese Academy of Sciences, Beijing 100049, People's Republic of China
Japan, and South Korea (Chinese Higher Fungi: 18 volumes). It was first recorded in the Shennong's Classic of Meteria Medica, and classified as an upper-grade medicine in medical books [2]. About 78 species of Ganoderma are recorded in Chinese Higher Fungi, of which, G. lucidum and $G$. sinense, were found to be edible and medicinallybeneficial fungi, and were registered in Chinese Pharmacopoeia (2010 and 2015 edition). However, other species, such as G. capense, G. cochlear, and G. tsuage, also play an important part in traditional folk medicines. In addition, pharmacological studies have also involved the extract and chemical constituents of other species [3-5]. Until now, the 
chemical constituents and biological activities of 22 species of Ganoderma have been studied.

Ganoderma is rich in novel "mycochemicals", including polysaccharide, triterpenoids, steroids, fatty acids, etc. Although polysaccharide is found to be one of the main bioactive constituents, its high molecular weight and complex structure limits its use in the drug market. Meanwhile, the small molecular constituents have played a significant role over the last 200 years in treating and preventing diseases, and are continuing to serve as important leads in modern drug discovery [6-11].

Since the discovery of ganomycins A and B [12], more than 100 aromatic meroterpenoids, derived by a hybrid of shikimic acid and mevalonic acid biogenetical pathway, were isolated from the genus Ganoderma (Ganodermataceae) [13]. Ganoderma meroterpenoids (GMs) have attracted increasing attention because they showed diverse structural skeletons and series of bioactivities, such as NO production inhibitory [14], anti-oxidant [15, 16], anti-allergic [17, 18], anti-fibrotic [19], anti-Acetyl cholinesterase (AChE) [20], cytotoxic [21], antimicrobial [12], and aldose reductase inhibitory activities [22]. As a result, chemists have synthesized polycyclic meroterpenoids by employing many steps [23-26].

Herein, we review the structure, bioactivities, and biosynthesis pathways of GMs from Ganoderma species to lay the foundation for the further research and provide the important sources for the development of lead compounds.

\section{Biosynthetic Pathway of GMs}

The prenylation of aromatic compounds plays an important role in the natural product research because it not only gives rise to an astounding diversity of small molecular constituents in plants, fungi and bacteria, but also enhances the bioactivities and bioavailabilities of these compounds [27]. Aromatic prenyltransferase is the key enzyme for the prenylation of aromatic compounds. Meroterpenoids including ubiquinone, plastoquinone, menadione, vitamin E, prenylflavonoids, shikonin and prenylated alkaloids, are formed under prenyltransferase [28]. The analysis of the genome showed that abundant carbohydrate-active enzymes and ligninolytic enzymes were present in the $G$. lucidum genome [29]. All the meroterpenoids from Ganoderma consist of a 1,2,4-trisubstituted phenyl group and a polyunstaturated terpenoid parts, suggesting that lignin was degraded to phenyl group by the liginolytic enzymes of Ganoderma, and the terpenoid parts were further assembled under prenyltransferase.

\section{Chemical Structures and Bioactivities of GMs}

A class of GMs, which had a 1,2,4-trisubtituted phenyl group connecting with $\mathrm{C} 10$ or $\mathrm{C} 15$ polyunsaturated side chain or polycyclic substructure, widely distributed in genus Ganoderma. According to the difference in their terpenoid parts, these GMs can be divided into three types.

\subsection{Chain-Contained GMs}

Due to the presence of double bonds in terpenoid part, the redox reaction can take place in allylic position (Fig. 1, Table 1). Thus, compounds 1-6, and 9-13 had a ketone carbonyl at $\mathrm{C}-1^{\prime}$ and a carboxyl or methyl ester at C-10' or C-14' [15, 16, 30-35]. Among them, compounds 2 and 13 existed positional isomerization of olefinic bond because of the shift of the double bond at C- $2^{\prime}$ and C- $3^{\prime}[30,35]$, whereas, the reduction of the $\Delta^{21,31}$ in chizhine $\mathrm{D}(\mathbf{3})$, cochlearin $\mathrm{G}(\mathbf{4})$, applanatumols $\mathrm{S}, \mathrm{T}(\mathbf{5}, \mathbf{6})$ and ganomycin E (9) was occurred [30, 31, 34]. The C-14' of ganomycin F (7) was connected to a hydroxyl group [16]. The trans $-\Delta^{2^{\prime}, 3^{\prime}}$ of ganoleucin B (8) was isomerized to cis under conditions of enzyme or light [33]. The $\Delta^{10^{\prime}, 11^{\prime}}$ of ganomycin J (9) was oxidized to two hydroxyls. Fornicin D (1), cochlearins H, G, I $(\mathbf{2}, \mathbf{4}, \mathbf{1 2})$ and ganomycin C (11) isolated from Ganoderma cochlear, as well as ganomycins $\mathrm{F}$ and $\mathrm{E}$ (7 and 10) gained from G. capense, showed significant anti-oxidant activities $[15,16,30]$. Compound $\mathbf{3}$ was isolated from G. lucidum and displayed weak renoprotective effect [31]. The biological assay of applanatumols $\mathrm{S}$ and $\mathrm{T}(\mathbf{5}, \mathbf{6})$ from $G$. applanatum [32], and ganoleucin B (8) from G. leucocontextum didn't show inhibitory activities against COX-1, COX-2, HMG-CoA reductase and $\alpha$-glucosidase, respectively [33]. However, ganomycin J (9) from G. lucidum showed strong inhibitory activity against $\mathrm{HMG}-\mathrm{CoA}$ reductase with an $\mathrm{IC}_{50}$ value of $30.3 \mu \mathrm{M}[34]$.

An $\alpha, \beta$-unsaturated $\gamma$-lactone fraction can be formed through a nucleophilic reaction from the carboxyl at C-10' or $\mathrm{C}-14^{\prime}$ to the ketone carbonyl at $\mathrm{C}-1^{\prime}$ (Fig. 2, Table 1). Cao et al [37] investigated the fruiting bodies of $G$. sinense and a series of GMs with an $\alpha, \beta$-unsaturated $\gamma$-lactone fraction, namely $(+)$-zizhines A-F $(\mathbf{1 7}, \mathbf{2 0}-\mathbf{2 4})$, were isolated. All the compounds were evaluated for their inhibition on extracellular matrix component (fibronectin) generation by using TGF- $\beta 1$ induced rat kidney tubular epithelial cells. However, all of them didn't show any inhibitory activities. $( \pm)$-Chizhine $\mathrm{E}$ and $\mathrm{F}(\mathbf{1 5}, \mathbf{1 9})$ and ( \pm )-lucidulactone (16) were isolated from G. lucidum and the individual enantiomers of compounds $\mathbf{1 5}$ and $\mathbf{1 9}$ significantly inhibit monocyte chemotactic protein 1 (MCP-1) 
<smiles>[R]C(C)=CCCC(CC(=O)c1cc(O)ccc1O)C(=O)O[As](C)(=O)C(=O)O</smiles>
Fornicin D (1)

Cochlearin H (2)

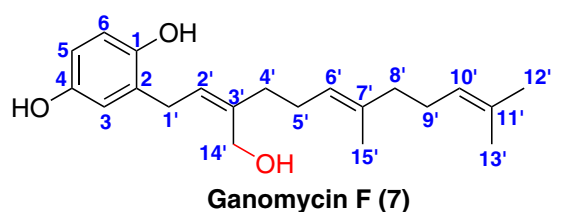<smiles>CC(C=O)=CCC/C(C)=C/CCCC(CC(=O)c1cc(O)ccc1O)C(=O)OC(C)C</smiles><smiles>CC(C)=CCCC(C)=CCCC(=CCc1cc(O)ccc1O)C(=O)O</smiles><smiles>C/C(=C\CC/C(=C/Cc1cc(O)ccc1O)C(=O)O)CC[C@@H](O)C(C)(C)O</smiles>

Ganoleucin B (8)<smiles>CC(C)=CCC/C(C)=C/C/C=C(/CC(=O)c1cc(O)ccc1O)C(=O)O</smiles>

Cochlearol D (13)

Fig. 1 Structures of GMs with a 10-carbon or 15-carbon chain

and fibronectin production in a dose-dependent manner [31, 36]. Fornicin E (25) obtained from G. capense also was a pair of enantiomers, which showed stronger DPPH scavenging activity than vitamin $\mathrm{E}$ (positive control) [16]. ( \pm )-Applanatumol $U$ (14) was identified from $G$. applanatum and showed no inhibition against COX-1 and COX-2 [32].

Three pairs of enatiomers (Fig. 3, Table 1), ( \pm )chizhines A-C (26-28) possessing a $\left(6^{\prime} \rightarrow 10^{\prime}\right)$ - $\gamma$-lactone ring and a $\left(7^{\prime} \rightarrow 10^{\prime}\right)$ - $\delta$-lactone ring, respectively, were isolated from the fruiting bodies of G. lucidum. These compounds showed weak renoprotective effects [31].

With the help of oxidases, the ether ring was present in many GMs (Fig. 4, Table 1). For example, compounds 2935 had different ether ring in the terpenoid part, whereas, the ether rings in compounds $\mathbf{3 6}-\mathbf{4 0}$ were formed through a cyclization between the hydroxyl at $\mathrm{C}-1$ and the hydroxyls of the terpenoid part. Compounds $\mathbf{2 9}, \mathbf{3 0}$, and 35-38 displayed significant antioxidant activities in the DPPH scavenging assay $[16,30]$. Among them, $( \pm)$-cochlearin $D$ (30) and (+)-30 exhibited weak inhibitory effects for the proliferation of hepatic stellate cells (HSCs) induced by transforming growth factor- $\beta 1$ (TGF- $\beta 1$ ) [30]. Except for above compounds, the rest of compounds didn't show renoprotective activities $[32,33]$.

\subsection{Polycyclic GMs}

Because of the presence of polyunsaturated terpenoid part, free radical reaction can be occurred in GMs under the conditions of enzyme and light, which led to the formation of polycyclic structures (Table 2).

Compounds 41-58 (Fig. 5) were derived from the biogenetic precusor fornicin D (1), of which compounds 41$\mathbf{4 6}$ had a five-membered carbon ring in the terpenoid part through the connection between $\mathrm{C}-2^{\prime}$ and $\mathrm{C}-6^{\prime}$ [32]; wheares, compounds 47-57 possessed a six-membered carbon ring by a linkage between $\mathrm{C}-3^{\prime}$ and $\mathrm{C}-9^{\prime}$ [32, 38-41]. The presence of a seven-membered carbon ring in compound $\mathbf{5 8}$ was formed due to the carbon bond at $\mathrm{C}-2^{\prime}$ and C-9' [38]. The inhibitory activities against COX-1 and COX-2 of compounds $\mathbf{4 1 - 5 6}$ were evaluated and they didn't show obvious inhibition [32, 39-41]. Compound $\mathbf{5 7}$ was found to promote proliferation of neural stem cells (NSCs) [38]. However, compound $\mathbf{5 8}$ can inhibit NSC proliferation compared with a DMSO control [38].

When ganomycin $\mathrm{C}$ (11) was the biosynthetic precusor, compounds 59-70 (Fig. 5) were formed through the cyclization between $\mathrm{C}^{-} \mathbf{6}^{\prime}$ and $\mathrm{C}-10^{\prime}$ [30, 40]. Biological activity of all the GMs against COX-2 was evaluated in vitro, only ganotheaecoloid $\mathbf{J}(\mathbf{6 8})$ was found to have COX-2 inhibitory activity with an $\mathrm{IC}_{50}$ value of $9.96 \mu \mathrm{M}$ [40]. Cochlearin A (70) showing DPPH scavenging activity had a cyclohexane fraction, which was formed by $\mathrm{C}-1^{\prime}$ binding with $\mathrm{C}-6^{\prime}[30]$.

Furthermore, compounds bearing seven-membered carbon ring or five-membered carbon ring were as the precursor, the formation of an ether bond between C-1 and $\mathrm{C}-2^{\prime}$ resulted in the occurrence of sipro ring. For instance, compounds 71-80 (Fig. 6) contained a 6/5/7 ring system 
Table 1 Name, source and their bioactivities of chain-containing GMs

\begin{tabular}{|c|c|c|c|c|}
\hline Number & Name & Bioactivity & Source & Reference \\
\hline 1 & Fornicin D & Antioxidant activity & G. cochlear & {$[15]$} \\
\hline 2 & Cochlearin $\mathrm{H}$ & Antioxidant activity & G. cochlear & {$[30]$} \\
\hline 3 & Chizhine D & Renoprotective effect & G. lucidum & {$[31]$} \\
\hline 4 & Cochlearin G & Antioxidant activity & G. cochlear & {$[30]$} \\
\hline 5 & Applanatumol S & Inhibitory activities against $\mathrm{COX}-1, \mathrm{COX}-2$ & G. applanatum & {$[32]$} \\
\hline 6 & Applanatumol T & Inhibitory activities against $\mathrm{COX}-1, \mathrm{COX}-2$ & G. applanatum & {$[32]$} \\
\hline 7 & Ganomycin F & Antioxidant activity & G. capense & [15] \\
\hline 8 & Ganoleucin B & Inhibitory activities against HMG-CoA reductase and $\alpha$-glucosidase & $\begin{array}{l}\text { G. } \\
\text { leucocontextum }\end{array}$ & [33] \\
\hline 9 & Ganomycin J & $\begin{array}{l}\text { Inhibitory activity against } \mathrm{HMGs} \text { reductase }\left(\mathrm{IC}_{50}: 30 \mu \mathrm{M}\right) \text {, aldose reductase and } \\
\alpha \text {-glucosidase }\end{array}$ & G. lucidum & {$[34]$} \\
\hline 10 & Ganomycin E & DPPH radical scavenging activity & G. capense & {$[16]$} \\
\hline 11 & Ganomycin C & Antioxidant activity & G. cochlear & {$[15]$} \\
\hline 12 & Cochlearin I & DPPH radical scavenging & G. cochlear & {$[30]$} \\
\hline 13 & Cochlearol D & & G. cochlear & [35] \\
\hline 14 & $\begin{array}{l}(+)- \\
\text { Applanatumol } \\
\text { U }\end{array}$ & Inhibitory activity against $\mathrm{COX}-1$ and $\mathrm{COX}-2$ & G. applanatum & [32] \\
\hline 15 & $(+)$-Chizhine E & Renoprotective effects & G. lucidum & {$[31]$} \\
\hline 16 & $\begin{array}{l}(+)- \\
\text { Lucidulactone } \\
\text { B }\end{array}$ & & G. lucidum & [36] \\
\hline 17 & $(+)$-Zizhine A & Renoprotective effects & G. sinense & {$[37]$} \\
\hline 18 & $\begin{array}{l}(+) \text {-Ganoleucin } \\
\quad \mathrm{C}\end{array}$ & Inhibition against HMG-CoA reductase and a-glucosidase & $\begin{array}{l}\text { G. } \\
\quad \text { leucocontextum }\end{array}$ & {$[33]$} \\
\hline 19 & $(+)$-Chizhine F & Renoprotective effects & G. lucidum & {$[31]$} \\
\hline 20 & $(+)$-Zizhine B & Renoprotective effects & G. sinense & {$[37]$} \\
\hline 21 & $(+)$-Zizhine $\mathrm{C}$ & Renoprotective effects & G. sinense & [37] \\
\hline 22 & $(+)$-Zizhine D & Renoprotective effects & G. sinense & [37] \\
\hline 23 & $(+)$-Zizhine E & Renoprotective effects & G. sinense & {$[37]$} \\
\hline 24 & $(+)$-Zizhine F & Renoprotective effects & G. sinense & {$[37]$} \\
\hline 25 & $(+)$-Fornicin E & Renoprotective effects & G. cochlear & [16] \\
\hline 26 & Chizhine A & Renoprotective effects & G. lucidum & {$[31]$} \\
\hline 27 & Chizhine B & Renoprotective effects & G. lucidum & {$[31]$} \\
\hline 28 & Chizhine $\mathrm{C}$ & Renoprotective effects & G. lucidum & {$[31]$} \\
\hline 29 & $(+)$-Cochlearin B & Antioxidant activity & G. cochlear & {$[30]$} \\
\hline 30 & $( \pm)$-Cochlearin D & Antioxidant activity & G. cochlear & {$[30]$} \\
\hline 31 & (+)-Lingzhine E & Neural stem cell proliferation & G. lucidum & {$[38]$} \\
\hline 32 & $\begin{array}{l}(+)- \\
\text { Applanatumol } \\
\text { P }\end{array}$ & Inhibitory activity against $\mathrm{COX}-1$ and $\mathrm{COX}-2$ & G. applanatum & {$[32]$} \\
\hline 33 & $\begin{array}{l}(+)- \\
\text { Applanatumol } \\
\text { Q }\end{array}$ & Inhibitory activity against $\mathrm{COX}-1$ and $\mathrm{COX}-2$ & G. applanatum & {$[32]$} \\
\hline 34 & $\begin{array}{l}(+)- \\
\text { Applanatumol } \\
\text { R }\end{array}$ & Inhibitory activity against $\mathrm{COX}-1$ and $\mathrm{COX}-2$ & G. applanatum & {$[32]$} \\
\hline 35 & $\begin{array}{l}( \pm)- \\
\text { Ganocapensin } \\
\text { A }\end{array}$ & Inhibitory activity against $\mathrm{COX}-1$ and $\mathrm{COX}-2$ & G. capense & {$[16]$} \\
\hline 36 & Ganocapensin B & Antioxidant activity & G. capense & {$[16]$} \\
\hline
\end{tabular}


Table 1 (continued)

\begin{tabular}{lllll}
\hline Number & Name & Bioactivity & Source & Reference \\
\hline $\mathbf{3 7}$ & ( \pm -Cochlearin E & Antioxidant activity & G. cochlear & {$[30]$} \\
$\mathbf{3 8}$ & Cochelarin F & Antioxidant activity & G. cochlear & {$[30]$} \\
$\mathbf{3 9}$ & Applanatumol Z1 & Inhibitory activity against COX-1 and COX-2 & G. applanatum & {$[32]$} \\
$\mathbf{4 0}$ & Cochlearol C & & G. cochlear & {$[33]$} \\
\hline
\end{tabular}<smiles>C/C(=C\CCC1=CC(c2cc(O)ccc2O)OC1=O)CO</smiles>

(+)-Applanatumol U (14)<smiles>C/C(C=O)=C\CCC1=CC(c2cc(O)ccc2O)OC1=O</smiles>

(+)-Chizhine E (15)<smiles>COC1(c2cc(O)ccc2O)OC(=O)C(CC/C=C(\C)C=O)O1</smiles>

(+)-Lucidulactone B (16)<smiles>C/C(=C\CC/C(C)=C/CC/C=C(\C)CC/C=C(\C)CCCC1=C[C@@H](c2cc(O)ccc2O)OC1=O)CO</smiles>

(+)-Zizhine A (17)

(+)-Ganoleucin C (18)<smiles>CC(C)=CC(=O)CC(C)=CCCC1=CC(c2cc(O)ccc2O)OC1=O</smiles>

(+)-Chizhine F (19)<smiles>CC(=O)OC/C(C)=C/CC/C(=C/CCC1=C[C@@H](c2cc(O)ccc2O)OC1=O)CC/C=C(\C)CO</smiles>

(+)-Zizhine B (20)

(+)-Zizhine C (21)

(+)-Zizhine D (22)<smiles>CC(=O)OC/C(C)=C/CCC(CO)CCCC1=CC(c2cc(O)ccc2O)OC1=O</smiles>

(+)-Zizhine E (23)<smiles>COC1(c2cc(O)ccc2O)C=C(CC/C=C(\CO)CC/C=C(\C)COC(C)=O)C(=O)O1</smiles><smiles>COC1(c2cc(O)ccc2O)C=C(CC/C=C(\C)CC/C=C(\C)CO)C(=O)O1</smiles>

(+)-Fornicin E (25)<smiles>CC(C)=C[C@H]1C[C@@H](CC(=O)c2cc(O)ccc2O)C(=O)O1</smiles>

Chizhine A (+)-26: 3'S, 6'S<smiles>CC(C)=C[C@@H]1C[C@@H](CC(=O)c2cc(O)ccc2O)C(=O)O1</smiles>

Chizhine B (+)-27: 3'R, 6'S<smiles>C=C(C)[C]1CC[C@@](O)(CC(=O)c2cc(O)ccc2O)C(=O)O1</smiles>

Chizhine C (+)-28: 3'R, 7'R

Fig. 3 Structures of GMs with a $\left(6^{\prime} \rightarrow 10^{\prime}\right)$ - or $\left(7^{\prime} \rightarrow 10^{\prime}\right)$-lactone

[42] and compounds 81-92 (Fig. 6) possessed a 6/5/5 ring system [38, 42]. Biological evaluation disclosed that compounds $\mathbf{7 7}$ and $\mathbf{7 8}$ inhibited JAK3 kinase with $\mathrm{IC}_{50}$ values of $7.0 \pm 3.2$ and $34.8 \pm 21.1 \mu \mathrm{M}$, respectively [42]. The most potent member of this series, (-)-spirolingzhine A (89), was shown to affect NSC cell cycle progression using the 5-bromo-2-deoxyuridine (BrdU) incorporation assay [38].

A series of bridge-ring compounds were formed through the free radical reactions. The structures of compounds 93 105 (Fig. 7) had a five-membered carbon ring fraction fusing with a $\gamma$-lactone ring [15, 32, 43]. Among them, 


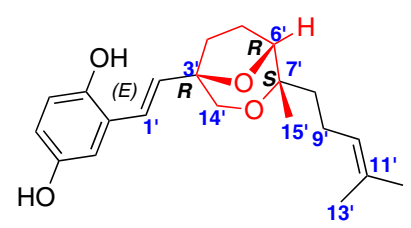

(+)-Cochlearin B (29)<smiles>CC(C)=CCC/C(C)=C/C/C=C1/C[C@@H](c2cc(O)ccc2O)O[C@@H]1C(=O)C[13C](=O)c1cc(O)ccc1O</smiles>

( \pm )-Cochlearin D (30)

(+)-Lingzhine E (31)

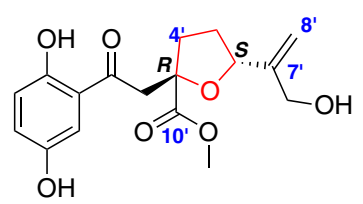

(+)-Applanatumol P (32)<smiles>CC(CO)[C@H]1CC[C@@](CC(=O)c2cc(O)ccc2O)(C(=O)O)O1</smiles>

(+)-Applanatumol Q (33): $\mathrm{R}=\mathrm{CH}_{3}$ (+)-Applanatumol R (34): $\mathbf{R = H}$

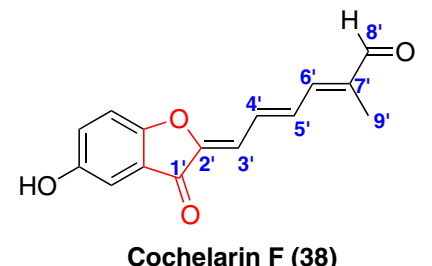

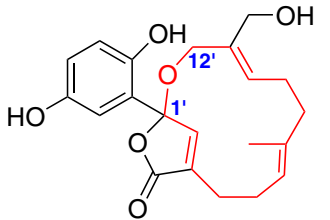

( \pm )-Ganocapensin A (35)<smiles>COC(=O)CCc1cc(=O)c2cc(O)ccc2o1</smiles>

Applanatumol Z1 (39)<smiles>CC(C)CCCC1=CCCC(C(=O)O)=C1Cc1cc(O)ccc1OC(C)(C)C</smiles>

Ganocapensin B (36)

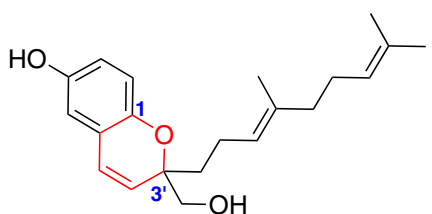

( \pm )-Cochlearin E (37)<smiles>CC(C)=CCC/C(C)=C/CCc1oc2ccc(O)cc2c(=O)c1O</smiles>

Cochlearol C (40)

Fig. 4 Structures of GMs with an ether ring

ganoderin A (93) disclosed significant antioxdiant activities [15]. In the bioassay, compounds 94-100 didn't exhibit inhibition aganist COX-1 and COX-2 [32]. The in vitro and in vivo results suggested that lingzhilactone B (102) could protect against renal injuries by increasing the activities of antioxidants and inhibiting inflammation [43]. The inhibition of Smad3 phosphorylation suggested that this substance displays in vivo antifibrotic activity by a mechanism that is dependent on disruption of Smad3. Applanatumol C (106) and linzhiol (107) beared an unusual 5/5/6/6 ring systerm characteristic of sharing a C- $3^{\prime}-\mathrm{C}-7^{\prime}$ axis (Fig. 7) $[32,44]$. The mirror of compound $\mathbf{1 0 6}$ was found to have COX-2 inhibitory effect with $\mathrm{IC}_{50}$ value of $25.5 \mathrm{mM}$ [32]. $(+)$-Lingzhiol (107) and (-)-lingzhiol (107) could selectively inhibit the phosphorylation of Smad3 in TGF- $\beta 1$ induced rat renal proximal tubular cells and activate Nrf2/ Keap1 in mesangial cells under diabetic conditions [44].

Cochlearin A (70) was as the biogenetic precursor for compounds 108-112 (Fig. 7) with an additional ether bond $\left(\mathrm{C}-1-\mathrm{C}-7^{\prime}\right)[15,38]$. The further cyclization led to the formation of ganoderins A-C (113-115) (Fig. 7) possessing a spiro[4,5]decane ring system, along with ganocin D (116) (Fig. 7) with an eight-membered ring [45]. Similarly, compounds 108-112 showed comparable antioxidant effects compared to the positive control (Vitamin E) [15, 45], while compounds 113-116 (Fig. 7) displayed anti-BuChE activities [45]. Cochlearol A (117) was a new normeroterpenoid containing a naturally unusual dioxaspiro[4.5]decane motif [46]. Compound 118 (Fig. 7) was a novel meroterpenoid possessing respective $4 / 5 / 6 / 6 / 6$ polycyclic ring systems [46]. Meanwhile, biological studies showed that (-)-118 was a strong inhibitor of pSmads, exhibiting renoprotective activities in TGF- $\beta 1$ induced rat renal proximal tubular cells [46]. Applanatumols A (120) and B [(士)-121] (Fig. 7) possessed a novel spiro[benzofuran-2,2'-biocyclo[3.2.2]nonane] ring system and a naturally unusual dioxacyclopenta[ $c d]$ inden motif, respectively [18]. Both of them didn't show inhibitory activities against renal fibrosis in rat proximal tubular epithelial cells [18].

\subsection{Dimeric GMs}

Except for the intramolecular cyclization, the intermolecular cyclization was present in GMs, which resulted in the formation of dimeric GMs (Fig. 8, Table 3). (+)- and (-)siensilactam A (121) was a novel hybrid metabolites possessing a unique $2 H$-pyrrolo[2,1-b][1,3]oxaz-in-6(7H)-one ring system [47]. (-)-121 was found to be a Smad3 phosphorylation inhibitor in TGF- $\beta 1$ induced human renal proximal tubular cells [47]. ( \pm )-Ganoapplanin (122) feartured an unprecedented dioxaspirocyclic skeleton, which was constructed from a 2,4-dihydroxy benzoic acid and a bridge-ring compound 102 [48]. Biological studies showed that $( \pm)-122$ and its enantomers exhibited different inhibitory activities on T-tpye voltage-gated calcium channels [48]. Applanatumin A (123) possessed a new hexacyclic skeleton containing spro[benzofuran-2,1'-cyclopentane] motif [17]. The analysis of its sturcture showed that it 
Table 2 Name, source and bioactivities of polycyclic GMs

\begin{tabular}{|c|c|c|c|c|}
\hline Number & Name & Bioactivity & Source & References \\
\hline 41 & Applanatumol V & Inhibitory activities against $\mathrm{COX}-1$ and $\mathrm{COX}-2$ & G. applanatum & {$[32]$} \\
\hline 42 & Applanatumol W & Inhibitory activities against $\mathrm{COX}-1$ and $\mathrm{COX}-2$ & G. applanatum & {$[32]$} \\
\hline 43 & Applanatumol X & Inhibitory activities against $\mathrm{COX}-1$ and $\mathrm{COX}-2$ & G. applanatum & {$[32]$} \\
\hline 44 & Applanatumol Y & Inhibitory activities against $\mathrm{COX}-1$ and $\mathrm{COX}-2$ & G. applanatum & {$[32]$} \\
\hline 45 & Applanatumol Z & Inhibitory activities against COX-1, COX-2 & G. applanatum & {$[32]$} \\
\hline 46 & Applanatumol Z2 & Inhibitory activities against $\mathrm{COX}-1, \mathrm{COX}-2$ & G. applanatum & {$[32]$} \\
\hline 47 & Applanatumol K & Inhibitory activities against $\mathrm{COX}-1$ and $\mathrm{COX}-2$ & G. applanatum & {$[32]$} \\
\hline 48 & Applanatumol L & Inhibitory activities against $\mathrm{COX}-1$ and $\mathrm{COX}-2$ & G. applanatum & {$[32]$} \\
\hline 49 & Applanatumol M & Inhibitory activities against $\mathrm{COX}-1$ and $\mathrm{COX}-2$ & G. applanatum & {$[32]$} \\
\hline 50 & Applanatumol N & Inhibitory activities against $\mathrm{COX}-1$ and $\mathrm{COX}-2$ & G. applanatum & {$[32]$} \\
\hline 51 & Applanatumol O & Inhibitory activities against $\mathrm{COX}-1$ and $\mathrm{COX}-2$ & G. applanatum & {$[32]$} \\
\hline 52 & Chizhiol A & Inhibitory activities against $\mathrm{COX}-1$ and $\mathrm{COX}-2$ & G. lucidum & [39] \\
\hline 53 & Ganotheaecoloid L & Inhibitory activities against $\mathrm{COX}-1$ and $\mathrm{COX}-2$ & G. theaecolum & [40] \\
\hline 54 & (+)-Ganotheaecoloid M & Inhibitory activities against $\mathrm{COX}-1$ and $\mathrm{COX}-2$ & G. theaecolum & [40] \\
\hline 55 & (-)-Ganotheaecoloid N & Inhibitory activities against $\mathrm{COX}-1$ and $\mathrm{COX}-2$ & G. theaecoloum & [40] \\
\hline 56 & Petchiene A & Inhibitory activities against $\mathrm{COX}-1$ and $\mathrm{COX}-2$ & G. petchii & [41] \\
\hline 57 & Lingzhine $\mathrm{C}$ & Promote proliferation of neural stem cells (NSCs) & G. lucidum & {$[38]$} \\
\hline 58 & $( \pm)$-Lingzhine B & Inhibit NSC proliferation & G. lucidum & {$[38]$} \\
\hline 59 & (-)-Ganotheaecoloid A & Inhibitory activities against COX-2 & G. theaecolum & [40] \\
\hline 60 & (-)-Ganotheaecoloid B & Inhibitory activities against $\mathrm{COX}-2$ & G. theaecolum & [40] \\
\hline 61 & Ganotheaecoloid C & Inhibitory activities against $\mathrm{COX}-2$ & G. theaecolum & [40] \\
\hline 62 & Ganotheaecoloid D & Inhibitory activities against $\mathrm{COX}-2$ & G. theaecolum & [40] \\
\hline 63 & Ganotheaecoloid E & Inhibitory activities against COX-2 & G. theaecolum & [40] \\
\hline 64 & (-)-Ganotheaecoloid F & Inhibitory activities against $\mathrm{COX}-2$ & G. theaecolum & [40] \\
\hline 65 & Ganotheaecoloid G & Inhibitory activities against COX-2 & G. theaecolum & [40] \\
\hline 66 & Ganotheaecoloid H & Inhibitory activities against $\mathrm{COX}-2$ & G. theaecolum & [40] \\
\hline 67 & Ganotheaecoloid I & Inhibitory activities against $\mathrm{COX}-2$ & G. theaecolum & [40] \\
\hline 68 & $(+)$-Ganotheaecoloid $\mathbf{J}$ & COX-2 inhibitory activity $\left(\mathrm{IC}_{50}: 9.96 \mu \mathrm{M}\right)$ & G. theaecolum & [40] \\
\hline 69 & Ganotheaecoloid K & Inhibitory activities against $\mathrm{COX}-2$ & G. theaecolum & [40] \\
\hline 70 & $(+)$-Cochlearin A & Antioxidant activity & G. cochlear & {$[30]$} \\
\hline 71 & Spiroapplanatumine A & Inhibitory activities against JAK3 kinase & G. applanatum & [42] \\
\hline 72 & Spiroapplanatumine $\mathrm{C}$ & Inhibitory activities against JAK3 kinase & G. applanatum & [42] \\
\hline 73 & Spiroapplanatumine E & Inhibitory activities against JAK3 kinase & G. applanatum & [42] \\
\hline 74 & Spiroapplanatumine $\mathrm{G}$ & Inhibitory activities against JAK 3 kinase & G. applanatum & [42] \\
\hline 75 & Spiroapplanatumine I & Inhibitory activities against JAK3 kinase & G. applanatum & [42] \\
\hline 76 & Spiroapplanatumine B & Inhibitory activities against JAK3 kinase & G. applanatum & [42] \\
\hline 77 & Spiroapplanatumine D & Inhibitory activities against JAK3 kinase $\left(\mathrm{IC}_{50}: 7.0 \pm 3.2 \mu \mathrm{M}\right)$ & G. applanatum & [42] \\
\hline 78 & Spiroapplanatumine $\mathrm{F}$ & Inhibitory activities against JAK3 kinase ( IC $\left._{50}: 34.8 \pm 21.1 \mu \mathrm{M}\right)$ & G. applanatum & [42] \\
\hline 79 & Spiroapplanatumine $\mathrm{H}$ & Inhibitory activities against JAK3 kinase & G. applanatum & [42] \\
\hline 80 & Spiroapplanatumine $\mathrm{J}$ & Inhibitory activities against JAK3 kinase & G. applanatum & [42] \\
\hline 81 & Spiroapplanatumine $\mathrm{K}$ & Inhibitory activities against JAK3 kinase & G. applanatum & {$[42]$} \\
\hline 82 & Spiroapplanatumine L & Inhibitory activities against JAK 3 kinase & G. applanatum & [42] \\
\hline 83 & Spiroapplanatumine $\mathrm{M}$ & Inhibitory activities against JAK3 kinase & G. applanatum & [42] \\
\hline 84 & $(+)$-Spiroapplanatumine $\mathrm{N}$ & Inhibitory activity against JAK3 kinase & G. applanatum & {$[42]$} \\
\hline 85 & Spiroapplanatumine $\mathrm{O}$ & Inhibitory activities against JAK3 kinase & G. applanatum & {$[42]$} \\
\hline 86 & (-)-Spiroapplanatumine $\mathrm{N}$ & Inhibitory activities against JAK3 kinase & G. applanatum & {$[42]$} \\
\hline 87 & Spiroapplanatumine $\mathrm{P}$ & Inhibitory activities against JAK3 kinase & G. applanatum & {$[42]$} \\
\hline 88 & Spiroapplanatumine Q & Inhibitory activities against JAK3 kinase & G. applanatum & {$[42]$} \\
\hline
\end{tabular}


Table 2 (continued)

\begin{tabular}{|c|c|c|c|c|}
\hline Number & Name & Bioactivity & Source & References \\
\hline 89 & $(+)$ - Spirolingzhine A & Protective effects for NSC & G. lucidum & {$[38]$} \\
\hline 90 & $(+)$-Spirolingzhine B & Protective effects for NSC & G. lucidum & {$[38]$} \\
\hline 91 & $(+)$-Spirolingzhine $\mathrm{C}$ & Protective effects for NSC & G. lucidum & {$[38]$} \\
\hline 92 & Spirolingzhine D & Protective effects for NSC & G. lucidum & {$[38]$} \\
\hline 93 & $( \pm)$-Ganoderin A & Antioxidant activity & G. cochlear & [15] \\
\hline 94 & Applanatumol H & Inhibitory activities against $\mathrm{COX}-1, \mathrm{COX}-2$ & G. applanatum & {$[32]$} \\
\hline 95 & Applanatumol I & Inhibitory activities against $\mathrm{COX}-1, \mathrm{COX}-2$ & G. applanatum & {$[32]$} \\
\hline 96 & Applanatumol J & Inhibitory activities against $\mathrm{COX}-1, \mathrm{COX}-2$ & G. applanatum & {$[32]$} \\
\hline 97 & Applanatumol D & Inhibitory activities against $\mathrm{COX}-1, \mathrm{COX}-2$ & G. applanatum & {$[32]$} \\
\hline 98 & Applanatumol E & Inhibitory activities against $\mathrm{COX}-1, \mathrm{COX}-2$ & G. applanatum & {$[32]$} \\
\hline 99 & Applanatumol J & Inhibitory activities against $\mathrm{COX}-1, \mathrm{COX}-2$ & G. applanatum & {$[32]$} \\
\hline 100 & Applanatumol F & Inhibitory activities against $\mathrm{J} \mathrm{COX}-1, \mathrm{COX}-2$ & G. applanatum & {$[32]$} \\
\hline 101 & Lingzhilactone A & Renoprotective effect & G. lucidum & {$[43]$} \\
\hline 102 & Lingzhilactone B & Renoprotective effect & G. lucidum & [43] \\
\hline 103 & Lingzhilactone $\mathrm{C}$ & Renoprotective effect & G. lucidum & {$[43]$} \\
\hline 104 & Applanatumol Z3 & Inhibitory activities against JAK3 kinase & G. applanatum & {$[32]$} \\
\hline 105 & Applanatumol Z4 & Inhibitory activities against JAK3 and DDR1 kinases & G. applanatum & {$[32]$} \\
\hline 106 & Applanatumol C & Inhibitory activities against JAK3 and DDR1 kinases & G. applanatum & {$[32]$} \\
\hline 107 & (-)-Lingzhiol & Renoprotective effect & G. lucidum & {$[44]$} \\
\hline 108 & $( \pm)$-Ganocochlearin A & Antioxidant activity & G. cochlear & {$[15]$} \\
\hline 109 & $( \pm)$-Ganocochlearin B & Antioxidant activity & G. cochlear & {$[15]$} \\
\hline 110 & $( \pm)$-Ganocochlearin $\mathrm{C}$ & Antioxidant activity & G. cochlear & {$[15]$} \\
\hline 111 & $( \pm)$-Ganocochlearin D & Antioxidant activity & G. cochlear & {$[15]$} \\
\hline 112 & Lingzhine D & Anti-BuChE activity & G. lucidum & {$[38]$} \\
\hline 113 & $( \pm)$-Ganocin A & Anti-BuChE activity & G. cochlear & {$[45]$} \\
\hline 114 & $( \pm)$-Ganocin B & Anti-BuChE activity & G. cochlear & {$[45]$} \\
\hline 115 & $( \pm)$-Ganocin C & Anti-BuChE activity & G. cochlear & {$[45]$} \\
\hline 116 & $( \pm)$-Ganocin D & Anti-BuChE activity & G. cochlear & {$[45]$} \\
\hline 117 & Cochlearol A & Renoprotective effect & G. cochlear & {$[46]$} \\
\hline 118 & Cochlearol B & Renoprotective effect & G. cochlear & {$[46]$} \\
\hline 119 & Applanatumol A & Anti-renal fibrosis & G. applanatum & [18] \\
\hline 120 & $( \pm)$-Applanatumol B & Anti-renal fibrosis & G. applanatum & {$[18]$} \\
\hline
\end{tabular}

consisted of two meroterpenoid parts, sproapplanatumine $\mathrm{N}(\mathbf{8 4})$ and applanatumol S (5), which were connected by a key Diels-Alder reaction. In TGF- $\beta 1$-induced human renal proximal tubular cells, applanatumin A (123) diclosed potent antifibrotic activity [17]. Cochlearoids A-E (124128) containing a unique methanobenzo $[c]$ oxocino $[2,3,4-$ ij]-isochromene scafflod were also constructed by two meroterpenoids [49]. Among them, (+)-124, and (-)-126 significantly inhibited Cav3.1 TTCC and showed noticeable selectivity against Cav1.2, Cav2.1, Cav2.2 and Kv11.1
(hERG) channels [49]. The combination of two chiancontained GMs formed (+)-ganodilactone (129), cochlearoids $F$ and $G$ (130 and 131) [50, 51]. Similarly, when 2,4-dihydroy benzoic acid was linked with chaincontained GMs by the same method as ganoapplanin (124), compounds 132-135 were taken place. $( \pm)-,(+)-$, and $(-)$ ganodilactone (129) showed pancreatic lipase inhibitory activities and exhibited the $\mathrm{IC}_{50}$ values as $27.3,4.0$, and $2.5 \mu \mathrm{M}$, respectively [50]. In addition, other compounds were tested for their renoprotective activity against fibro- 


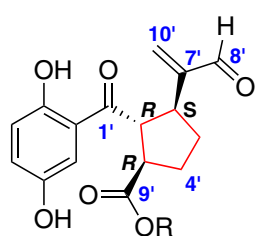

Applanatumol V (41): R=H Applanatumol W (42): $\mathrm{R}=\mathrm{CH}_{3}$<smiles>O=C(C[C@@]1(C(=O)O)C[Z](O)[C@@H](O)[C@@H]1O)c1cc(O)ccc1O</smiles>

Applanatumol K (47a): $\mathrm{R}=\mathrm{H}$ Applanatumol L (148a): $R=M e$<smiles>CC1=CCC[C@](CC(=O)c2cc(O)ccc2O)(C(=O)O)[C@H]1O</smiles>

Ganotheaecoloid L (53)<smiles>O=C(O)[C@H]1CC[C@@H]2Oc3ccc(O)cc3C(=O)[C@@H]21</smiles>

Applanatumol $X(43)$ : $R=H$ Applanatumol $Y$ (44): $R=M e$<smiles>C=C1OC(=O)[C@H](CC(=O)c2cc(O)ccc2O)[C@@H](O)C1=O</smiles>

Applanatumol M (49a) Applanatumol N (50a): $\mathrm{R}_{1}=\mathrm{OH}, \mathrm{R}_{2}=\mathrm{CH}_{2} \mathrm{OH}$ Applanatumol O (51a): $\mathbf{R}_{1}=\mathrm{H}, \mathrm{R}_{2}=\mathrm{CHO}$

Applanatumol Z (45)<smiles>[R]C1=CCC[C@@](CC(=O)c2cc(O)ccc2O)(C(=O)O)S1</smiles><smiles>COC(=O)[14C]1=[14CH]c2c1oc1ccc(O)cc1c2=O</smiles>

Applanatumol Z2 (46)<smiles>CC1=CCC[C@](CC(=O)c2cc(O)ccc2O)(C(=O)O)[C@H]1O</smiles>

Chizhiol A (52)<smiles>O=C[C+]1=CC=CC[C@@H](C(=O)c2cc(O)ccc2O)C1</smiles>

( \pm )-Lingzhine $B(58)$<smiles>O=C(C[14C]1(C(=O)O)CCC[C@@H](CO)[C@H]1O)c1cc(O)ccc1O</smiles>

(+)-Ganotheaecoloid M (54)<smiles>[R]C(=O)[C@@]1(CC(=O)c2cc(O)ccc2O)C=C(CO)CCC1</smiles>

(-)-Ganotheaecoloid N (55): $\mathrm{R}=\mathrm{OMe}$ Petchiene A (56): $\mathrm{R}=\mathrm{OH}$<smiles>O=Cc1cccc(CC(=O)c2cc(O)ccc2O)c1</smiles>

Lingzhine C (57)<smiles>C=C1CC[C@@H](O)C(C)(C)[C@@H]1CCCC1(O)Oc2ccc(O)cc2C1=O</smiles>

(-)-Ganotheaecoloid A (59)<smiles>CC1=CC[C@@H](O)C(C)(C)[C@@H]1CCCC1(O)Oc2ccc(O)cc2C1=O</smiles>

(-)-Ganotheaecoloid B (60)<smiles>C=C1CC[C@@H](O)C(C)(C)[C@@H]1C/C=C(/CC(=O)c1cc(O)ccc1O)C(=O)O</smiles>

Ganotheaecoloid D (62)<smiles>CC1=CC[C@@H](O)C(C)(C)[C@H]1CCC(CC(=O)c1cc(C)ccc1O)C(=O)O</smiles>

Ganotheaecoloid E (63)<smiles>CC1=CC[C@@H](O)C(C)(C)[C@H]1C/C=C(/CC(=O)c1cc(O)ccc1O)C(=O)O</smiles>

(-)-Ganotheaecoloid F (64)<smiles>C=C1CC[C@@H](O)C(C)(C)[C@@H]1CCC(CC(=O)c1cc(O)ccc1O)C(=O)O</smiles>

Ganotheaecoloid C (61)<smiles>CC1=CC[C@@H](O)C(C)(C)[C@@H]1CC/C(=C\C(=O)c1cc(O[13CH3])ccc1O)C(=O)O</smiles><smiles>C=C1CC[C@@H](O)C(C)(C)[C@H]1CCC1=CC(c2cc(O)ccc2O)OC1=O</smiles>

Ganotheaecoloid H (66)

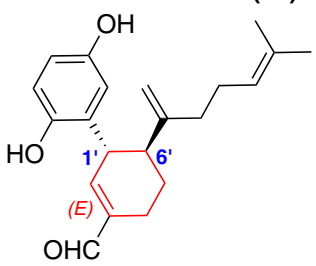

(+)-Cochlearin A (70)<smiles>C=C1CC[C@H](O)C(C)(C)[C@@H]1CCC1=CC(O)(c2cc(O)ccc2O)OC1=O</smiles>

(+)-Ganotheaecoloid J (68)<smiles>CC1(C)[C@H](O)CC[C@]2(C)OC(=O)C(CCc3cc(O)ccc3O)=CC[C@H]12</smiles>

Ganotheaecoloid K (69)

Fig. 5 Structures of GMs with a five-membered or six-membered carbon ring 


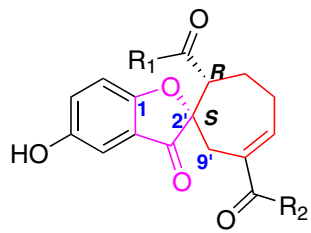

Spiroapplanatumine $A$ (71): $\mathbf{R}_{1}=\mathrm{OH}, \mathbf{R}_{2}=\mathrm{OH}$ Spiroapplanatumine C (72): $\mathrm{R}_{1}=\mathrm{OMe}, \mathrm{R}_{2}=\mathrm{OH}$ Spiroapplanatumine $E$ (73): $\mathbf{R}_{\mathbf{1}}=\mathrm{OH}, \mathrm{R}_{2}=\mathrm{OMe}$ Spiroapplanatumine $\mathrm{G}$ (74): $\mathrm{R}_{1}=\mathrm{OH}, \mathrm{R}_{2}=\mathrm{H}$ Spiroapplanatumine I (75): $\mathrm{R}_{1}=\mathrm{OMe}, \mathrm{R}_{2}=\mathrm{H}$

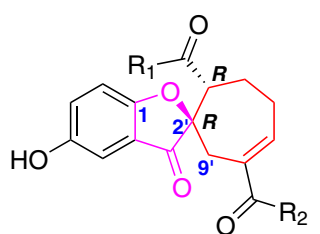

Spiroapplanatumine B (76): $\mathbf{R}_{\mathbf{1}}=\mathrm{OH}, \mathrm{R}_{\mathbf{2}}=\mathrm{OH}$ Spiroapplanatumine $D(77): \mathrm{R}_{1}=\mathrm{OMe}, \mathrm{R}_{2}=\mathrm{OH}$ Spiroapplanatumine $F(78): R_{1}=O H, R_{2}=O M e$ Spiroapplanatumine $H(79): R_{1}=O H, R_{2}=H$

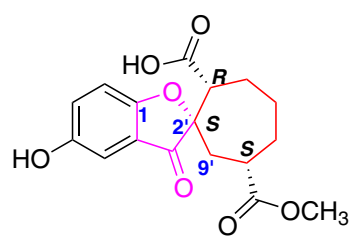

Spiroapplanatumine $\mathrm{J}(\mathbf{8 0})$<smiles>[R]C(=O)[C@@H]1CC[C@H](C(=C)CO)[C@@]12Oc1ccc(O)cc1C2=O</smiles><smiles>C=C(CO)C(=O)[C@]12Oc3ccc(O)cc3C(=O)[C@@]1(C(=O)O)CC[C@H]2C(=O)O</smiles><smiles>[R]C(=O)[C@H]1CC[C@H](C(=C)C=O)[C@@]12Oc1ccc(O)cc1C2=O</smiles>

Spiroapplanatumine K (81): R=OMe Spiroapplanatumine M (83) Spiroapplanatumine N (84a): R=OHSpiroapplanatumine N (86b) Spiroapplanatumine $\mathrm{L}$ (82): $\mathrm{R}=\mathrm{OH}$<smiles>COC(=O)[C@@H]1CC[C@@H](P(C)CO)[C@@]12Oc1ccc(O)cc1C2=O</smiles>

Spiroapplanatumine $\mathbf{P}(\mathbf{8 7})$

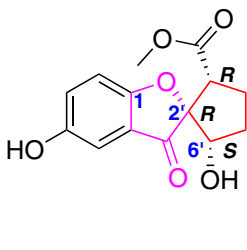

Spiroapplanatumine $Q(88)$<smiles>CC(CO)[C@H]1CCC(C(=O)O)[C@@]12Oc1ccc(O)cc1C2=O</smiles>

Spirolingzhine A (+)-89: Spirolingzhine C (+)-91 Spirolingzhine D (92) 2'S, 3'S, 6'R, 7'S Spirolingzhine $B(+)-90$ : 2'S, 3'S, 6'R, 7'R

Fig. 6 Structures of GMs with spiro ring

nectin inhibition in human proximal tubular epithelial cells (HKC-8). Compounds 130-133 and $\mathbf{1 3 5}$ exhibited potent inhibitory activity on fibronectin overproduction in TGF$\beta 1$-induced HKC-8 cells [51].

\section{Conclusion}

In this review, we summarized the chemical structures and biological activities of $135 \mathrm{GMs}$ in the last five years. Although the first GMs have been isolated in 2000, until recent years GMs were studied in-depth. Moreover, except for $G$. lucidum and $G$. sinense registered in Chinese Pharmacopoeia (2010 and 2015 edition), GMs were widely present in many other Ganoderma species, such as $G$. appalantum, G. capense, G. cochlear, and G. petchii. Above information indicated that GMs could play an important role in explaining the efficacy of Ganoderma. Thus, more bioactive studies should be carried out in the future for finding and developing lead compounds.

Furthermore, GMs possessed multiple prenyl groups or complex ring systems, which provided plentiful molecular model for various biological activities. However, we found that the majority of GMs showed racemic nature, which had impact on their bioactivites. Therefore, it is need to be separated using chiral HPLC method or be stereoselectively synthsized.

Addtionally, the formation of racemic GMs also attracted us attention. Analysis of these polycyclic GMs showed that their polycyclic structures are formed based on the polyunsaturated terpenoid fraction. Studies found that the cyclizations, such as cationic cyclization and radical cyclization, are the key factor to generate racemes. And these reactions can be taken place under conditions of acid, 


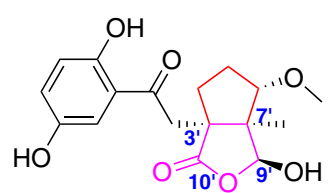

Ganoderin A (93)<smiles>COC(OC)[C@H]1COC(=O)[C@]2(CC(=O)c3cc(O)ccc3O)CC[C@@H](O)[C@H]12</smiles>

Applanatumol F (100)<smiles>C[C@@]12COC(=O)[C@]1(CC(=O)c1cc(O)ccc1O)CC[C@H]2O</smiles>

Applanatumols Z3 (104) and Z4 (105)

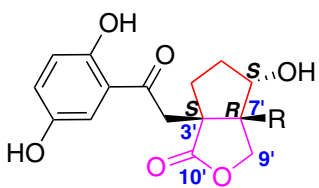

Applanatumol $\mathrm{H}$ (94): $\mathrm{R}=\mathrm{CH}_{2} \mathrm{OH}$ Applanatumol I (95): $\mathrm{R}=\mathrm{COOH}$ Applanatumol J (96): $\mathrm{R}=\mathrm{Cl}$

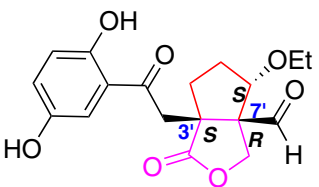

Lingzhilactone A (101)

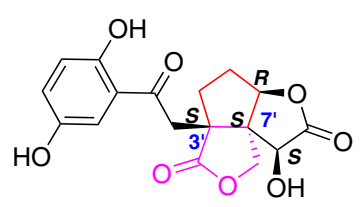

Applanatumol D (97)

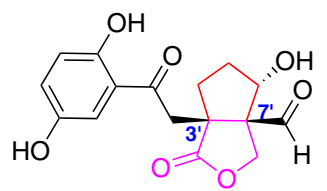

Lingzhilactone B (102)

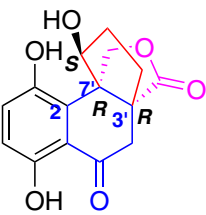

Applanatumol C (106)

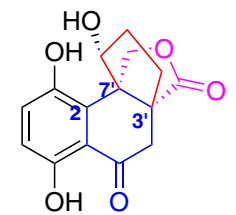

(-)-Lingzhiol (107)<smiles>COC(OC)[C@H]1COC(=O)[C@]2(CC(=O)c3cc(O)ccc3O)CC[C@@H]1CC2</smiles>

Applanatumol E (98): R=H Applanatumol $\mathrm{G}$ (99): $\mathrm{R}=\mathrm{Et}$

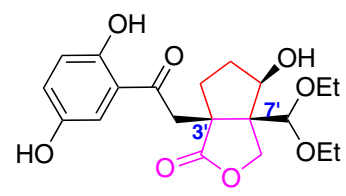

Lingzhilactone C (103)<smiles>CCCCCCCC1(C)Oc2ccc(O)cc2C2=CC(=O)CC[C@]21C</smiles>

(士)-Ganocochlearin A (108)<smiles>CC1(C)Oc2ccc(O)cc2C2=CC(=O)CC[C@H]21</smiles>

( \pm )-Ganocochlearin B (109)

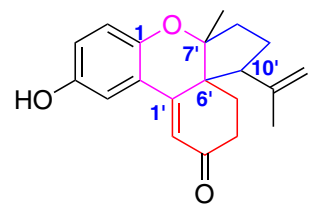

( \pm )-Ganocin B (114)

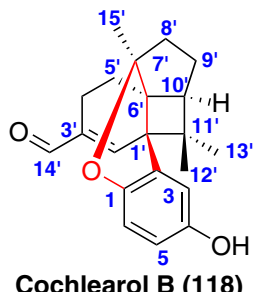<smiles>[R]c1ccc2c(c1)[C@@H]1[C@H]2c2cc(O)ccc2OC1(C)CCC=C(C)C</smiles>

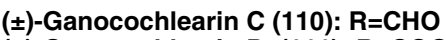
( \pm )-Ganocochlearin D (111): $\mathrm{R}=\mathrm{COOH}$<smiles></smiles>

( \pm -Ganocin B (115)

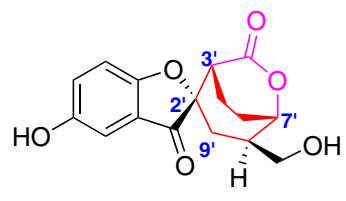

Applanatumol A (119)<smiles></smiles>

( \pm )-Ganocin B (116)
(+)-Applanatumol B (120)

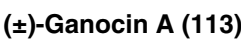<smiles>O=C1CCC2OC[C@@]3(O2)O[C@@](C(=O)O)(C1)c1c(O)ccc(O)c13</smiles>

Cochlearol A (117)

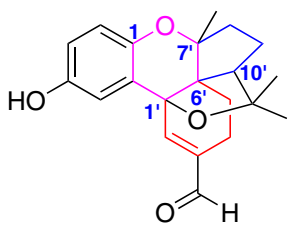

Fig. 7 Structures of GMs with bridge ring

light and heating. However, the reactions in the plants mostly involved in enzyme system, which led to the generation of stereoselective compounds. Thus, we deduced that these polycyclic GMs with racemic nature may be formed for defending high temperature, strong light and diseases.
In all, the efforts to discover novel GMs with interesting biological activity and intriguing strutures from Ganoderma species have long been a hot topic in natural products chemistry. Meanwhile, novel GMs will serve as an abundant resource for synthetic chemists. 
<smiles>O=C(C[C@@]12CCC3OC4CCC(=O)N4C(O)(OC1=O)[C@H]32)c1cc(O)ccc1O</smiles>

( \pm )-Sinensilactam A (121)

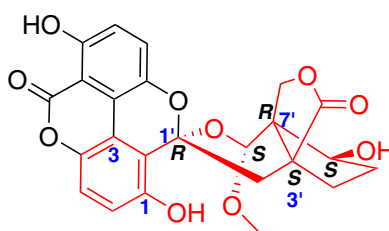

(+)-Ganoapplanin (122)

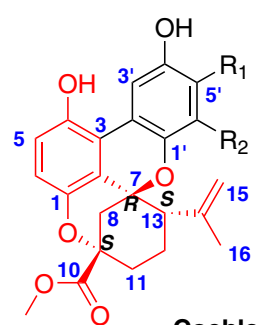<smiles>[R5]CC(=CCCC)CCC=C(C)CCC=C(C)C</smiles>

A<smiles>[B]C/C(=C\CCC)CC/C=C(/C)CC/C=C(/C)CCC=C(C)C</smiles>

Cochlearoid A (124): $R_{1}=H, R_{2}=A, R_{3}=O A C$

Cochlearoid $B$ (125): $R_{1}=H, R_{2}=A, R_{3}=H$

Cochlearoid C (126): $R_{1}=H, R_{2}=B, R_{3}=O H$

Cochlearoid D (127): $\mathbf{R}_{1}=\mathrm{B}, \mathrm{R}_{\mathbf{2}}=\mathrm{H}, \mathbf{R}_{\mathbf{3}}=\mathrm{OH}$

Cochlearoid E (128): $R_{1}=A, R_{2}=H, R_{3}=O A C$<smiles>[B]C(=CCC(C)C)CCC=C(C)CCC=C(C)C</smiles>

A<smiles>CC(C)=CCCC(=CCC(C)C)C(=O)O</smiles>

Cochlearoid F (130): $\mathrm{R}_{1}=\mathrm{H}, \mathrm{R}_{2}=\mathrm{A}, \mathrm{R}_{3}=\mathrm{CH}_{2} \mathrm{OH}$ Cochlearoid G (131): R1=A, R2=H, $\mathrm{R}_{3}=\mathrm{CH}_{2} \mathrm{OH}$<smiles>[R2]c1c(Br)c(O)cc2c(C(=O)c3cc(O)ccc3O)c(CCC=C(C)CCC=C(C)C)c(=O)oc12</smiles>
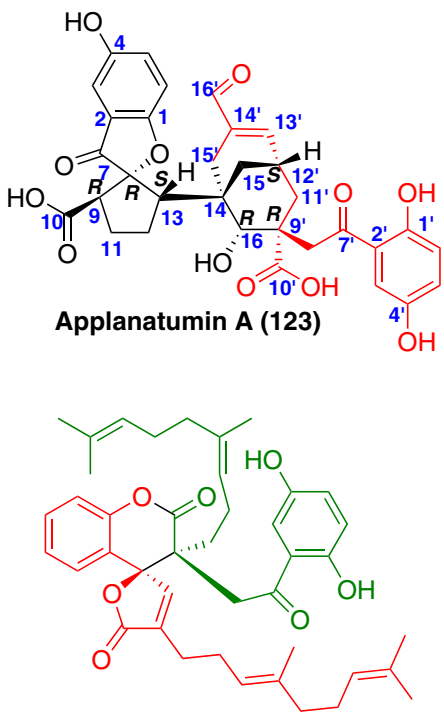

(+)-Ganodilactone (129)<smiles>[R2]c1c(Br)c(O)cc2c1oc(=O)c1c(O)ccc(O)c12</smiles>

Cochlearoid H (132): $\mathbf{R}_{\mathbf{1}}=\mathrm{H}, \mathbf{R}_{\mathbf{2}}=\mathrm{A}, \mathbf{R}_{\mathbf{3}}=\mathrm{COOH}$ Cochlearoid I (133): $\mathbf{R}_{1}=\mathrm{H}, \mathrm{R}_{2}=\mathrm{A}, \mathrm{R}_{3}=\mathrm{CH}_{2} \mathrm{OH}$ Cochlearoid J (134): $\mathrm{R}_{1}=\mathrm{A}, \mathrm{R}_{2}=\mathrm{H}, \mathrm{R}_{3}=\mathrm{CH}_{2} \mathrm{OH}$ Cochlearoid K (135): $R_{1}=B, R_{2}=H$

Fig. 8 Structures of dimeric GMs

Table 3 Name, source and bioactivities of dimeric GMs

\begin{tabular}{|c|c|c|c|c|}
\hline Number & Name & Bioactivity & Source & Reference \\
\hline 121 & $(-)$-Sinensilactam A & Renoprotective activity & G. sinense & [47] \\
\hline 122 & $(+)$-Ganoapplanin & Inhibitory activities on T-tpye voltage-gated calcium channels & G. applanatum & {$[48]$} \\
\hline 123 & Applanatumin A & Antifibrotic activity & G. applanatum & [17] \\
\hline 124 & $(-)$-Cochlearoid A & Inhibitory activities on T-tpye voltage-gated calcium channels & G. cochlear & [49] \\
\hline 125 & $(-)$-Cochlearoid B & Inhibitory activities on T-tpye voltage-gated calcium channels & G. cochlear & [49] \\
\hline 126 & (-)-Cochlearoid C & Inhibitory activities on T-tpye voltage-gated calcium channels & G. cochlear & [49] \\
\hline 127 & $(-)$-Cochlearoid D & Inhibitory activities on T-tpye voltage-gated calcium channels & G. cochlear & [49] \\
\hline 128 & $(-)$-Cochlearoid E & Inhibitory activities on T-tpye voltage-gated calcium channels & G. cochlear & [49] \\
\hline 129 & $(+)$-Ganodilactone & Inhibitory activity against pancreatic lipase & G. leucocontextum & {$[50]$} \\
\hline 130 & Cochlearoid F & Renoprotective effect & G. cochlear & {$[51]$} \\
\hline 131 & Cochlearoid G & Renoprotective effect & G. cochlear & {$[51]$} \\
\hline 132 & Cochlearoid $\mathrm{H}$ & Renoprotective effect & G. cochlear & [51] \\
\hline 133 & Cochlearoid I & Renoprotective effect & G. cochlear & {$[51]$} \\
\hline 134 & Cochlearoid J & Renoprotective effect & G. cochlear & {$[51]$} \\
\hline 135 & Cochlearoid K & Renoprotective effect & G. cochlear & {$[51]$} \\
\hline
\end{tabular}


Acknowledgements The research work was financially supported by the National Natural Science Foundation of China (No. 21702209 and No. 81172940), as well as Foundation of State Key Laboratory of Phytochemistry and Plant Resources in West China (P2010-ZZ14).

\section{Compliance with Ethical Standards}

Conflict of interest All authors declare no conflict of interest.

Open Access This article is distributed under the terms of the Creative Commons Attribution 4.0 International License (http://creative commons.org/licenses/by/4.0/), which permits unrestricted use, distribution, and reproduction in any medium, provided you give appropriate credit to the original author(s) and the source, provide a link to the Creative Commons license, and indicate if changes were made.

\section{References}

1. M. Upadhyay, B. Shrivastava, A. Jain, M. Kidwai, S. Kumar, J. Gomes, D. Goswami, A. Panda, R. Kuhad, Ann. Microbiol. 64, 839-846 (2014)

2. Y. Li, Z. Zhu, W. Yao, R. Chen, Med. Plant 3, 75-81 (2012)

3. S. Lee, S.H. Shim, J.S. Kim, K.H. Shin, S.S. Kang, Biol. Pharm. Bull. 28, 1103-1105 (2005)

4. P.H.K. Ngai, T.B. Ng, Biochem. Biophys. Res. Commun. 314, 988-993 (2004)

5. S. Gurunathan, J. Raman, S.N.A. Malek, P.A. John, S. Vikineswary, Int. J. Nanomed. 8, 4399-4413 (2013)

6. G.M. Cragg, D.J. Newman, K.M. Snader, J. Nat. Prod. 60, 52-60 (1997)

7. D.J. Newman, G.M. Cragg, K.M. Snader, Nat. Prod. Rep. 17, 215-234 (2000)

8. D.J. Newman, G.M. Cragg, K.M. Snader, J. Nat. Prod. 66, 1022-1037 (2003)

9. M.S. Butler, J. Nat. Prod. 67, 2141-2153 (2004)

10. I. Paterson, E.A. Anderson, Science 310, 451-453 (2005)

11. D.J. Newman, G.M. Cragg, J. Nat. Prod. 70, 461-477 (2007)

12. R.A.A. Mothana, R. Jansen, W.D. Julich, U. Lindequist, J. Nat. Prod. 63, 416-418 (2000)

13. X.Q. Chen, L.X. Chen, S.P. Li, J. Zhao, Phytochem. Lett. 22, 214-218 (2017)

14. M. Wang, F. Wang, F. Xu, Bioorg. Med. Chem. Lett. 26, 3342-3345 (2016)

15. X.R. Peng, J.Q. Liu, C.F. Wang, Z.H. Han, Y. Shu, X.Y. Li, L. Zhou, M.H. Qiu, Food Chem. 171, 251-257 (2015)

16. X.R. Peng, L. Li, X. Wang, G.L. Zhu, L. Zhou, M.H. Qiu, Fitoterapia 111, 18-23 (2016)

17. Q. Luo, L. Di, W.F. Dai, Q. Lu, Y.M. Yan, Z.L. Yang, R.T. Li, Y.X. Cheng, Org. Lett. 17, 1110-1113 (2015)

18. Q. Luo, L. Di, X.H. Yang, Y.X. Cheng, RSC Adv. 6, 45963-45967 (2016)

19. S.Z. Huang, B.H. Cheng, Q.Y. Ma, Q. Wang, F.D. Kong, H.F. Dai, S.Q. Qiu, P.Y. Zheng, Z.Q. Liu, Y.X. Zhao, RSC. Adv. 6, 21139-21147 (2016)

20. X.R. Peng, J.Q. Liu, Z.H. Han, X.X. Yuan, H.R. Luo, M.H. Qiu, Food Chem. 141, 920-926 (2013)

21. X.M. Niu, S.H. Li, H.D. Sun, C.T. Che, J. Nat. Prod. 69, 1364-1365 (2006)

22. J.J. Zhang, K. Ma, H.Y. Chen, K. Wang, W.P. Xiong, L. Bao, H.W. Liu, J. Antibiot. 70, 915-917 (2017)

23. K.S. Gautam, V.B. Birman, Org. Lett. 18, 1499-1501 (2016)
24. X.Y. Li, X.Y. Liu, X.Z. Jiao, H.G. Yang, Y.Y. Yao, P. Xie, Org. Lett. 18, 1944-1946 (2016)

25. D. Chen, H.M. Liu, M.M. Li, Y.M. Yan, W.D. Xu, X.N. Li, Y.X. Cheng, Chem. Commun. 51, 14594-14596 (2015)

26. H.X. Liu, L.Q. Hou, B. Yang, Y.F. Yuan, W.M. Zhang, Z.F. Xu, S.X. Qiu, H.B. Tan, Org. Lett. 19, 4786-4789 (2017)

27. X. Liu, R.D. Chen, D. Xie, J.H. Li, R.S. Wang, J.G. Dai, Yaoxue Xuebao 48, 161-169 (2013)

28. J. Gao, Y. Zeng, S. Lu, Zhiwu Xuebao (Beijing, China) 45, 751-759 (2010)

29. D. Liu, J. Gong, W. Dai, X. Kang, Z. Huang, H.M. Zhang, W. Liu, L. Liu, J. Ma, Z. Xia, Y. Chen, Y. Chen, D. Wang, P. Ni, A.Y. Guo, X. Xiong, PLoS ONE 7, e36146 (2012)

30. X.R. Peng, X. Wang, L. Chen, H. Yang, L. Lei, S.Y. Lu, L. Zhou, M.H. Qiu, Fitoterapia (2018). https://doi.org/10.1016/j.fitote. 2018.03.005

31. Q. Luo, X.L. Wang, L. Di, Y.M. Yan, Q. Lu, X.H. Yang, D.B. Hu, Y.X. Cheng, Tetrahedron 71, 840-845 (2015)

32. Q. Luo, X.H. Yang, Z.L. Yang, Z.C. Tu, Y.X. Cheng, Tetrahedron 30, 4564-4574 (2016)

33. K. Wang, L. Bao, K. Ma, J.J. Zhang, B.S. Chen, J.J. Han, J.W. Ren, H.J. Luo, H.W. Liu, Eur. J. Med. Chem. 127, 1035-1046 (2017)

34. M. Dou, R.T. Li, Y.X. Chen, Chin. Herb. Med. 8, 85-88 (2016)

35. B.S. Chen, J. Tian, J.J. Zhang, K. Wang, L. Liu, B. Yang, L. Bao, H.W. Liu, Fitoterapia 120, 6-16 (2017)

36. X.F. Wang, Y.M. Yan, X.L. Wang, X.J. Ma, X.Y. Fu, Y.X. Cheng, J. Asian Nat. Prod. Res. 17, 329-332 (2015)

37. W.W. Cao, Q. Luo, Y.X. Chen, S.M. Wang, Fitoterapia 110, 110-115 (2016)

38. Y.M. Yan, X.L. Wang, Q. Luo, L.P. Jiang, C.P. Yang, B. Hou, Z.L. Zuo, Y.B. Chen, Y.X. Cheng, Phytochemistry 114, 155-162 (2015)

39. F.J. Zhou, X.L. Wang, S.M. Wang, Y.X. Cheng, Nat. Prod. Res. Dev. 27, 22-25 (2015)

40. Q. Luo, Z.C. Tu, Z.L. Yang, Y.X. Cheng, Fitoterapia 125, 273-280 (2018)

41. Q.L. Gao, P.X. Guo, Q. Luo, H. Yan, Y.X. Cheng, Nat. Prod. Commun. 10, 2019-2022 (2015)

42. Q. Luo, X.Y. Wei, J. Yang, J.F. Luo, R. Liang, Z.C. Tu, Y.X. Cheng, J. Nat. Prod. 80, 61-70 (2017)

43. Y.M. Yan, X.L. Wang, L.L. Zhou, F.J. Zhou, R. Li, Y. Tian, Z.L. Zuo, P. Fang, A.C.K. Chung, F.F. Hou, Y.X. Cheng, J. Ethnopharm. 176, 385-393 (2015)

44. Y.M. Yan, J. Ai, L.L. Zhou, A.C.K. Chung, R. Li, J. Nie, P. Fang, X.L. Wang, J. Luo, Q. Hu, F.F. Hou, Y.X. Cheng, Org. Lett. 15, 5488-5491 (2013)

45. X.R. Peng, J.Q. Liu, L.S. Wan, X.N. Li, Y.Y. Yan, M.H. Qiu, Org. Lett. 16, 5262-5265 (2014)

46. M. Dou, L. Di, L.L. Zhou, Y.M. Yan, X.L. Wang, F.J. Zhou, Z.L. Yang, R.T. Li, F.F. Hou, Y.X. Cheng, Org. Lett. 16, 6064-6067 (2014)

47. Q. Luo, L. Tian, L. Di, Y.M. Yan, X.Y. Wei, X.F. Wang, Y.X. Cheng, Org. Lett. 17, 1565-1568 (2015)

48. L. Li, H. Li, X.R. Peng, B. Hou, M.Y. Yu, J.R. Dong, X.N. Li, L. Zhou, J. Yang, M.H. Qiu, Org. Lett. 18, 6078-6081 (2016)

49. F.J. Zhou, Y. Nian, Y.M. Yan, Y. Gong, Q. Luo, Y. Zhang, B. Hou, Z.L. Zuo, S.M. Wang, H.H. Jiang, J. Yang, Y.X. Cheng, Org. Lett. 17, 3082-3085 (2015)

50. H.P. Chen, Z.Z. Zhao, Y. Zhang, X. Bai, L. Zhang, J.K. Liu, RSC Adv. 6, 64469-64473 (2016)

51. X.L. Wang, F.J. Zhou, M. Dou, Y.M. Yan, S.M. Wang, L. Di, Y.X. Cheng, Bioorg. Med. Chem. Lett. 26, 5507-5512 (2016) 